1 Detrano R, Gianrossi R, Froelicher V. The diagnostic accuracy of the exercise electrocardiogram: a meta-analysis of 22 years of research. Prog Cardiovasc Dis 1989; 32: 173 206.

2 Fox RM, Hakki A, Iskandrian AS. Relation between electrocardiographic and scintigraphic location of myocardial ischemia during exercise in one-vessel coronary artery disease. Am 7 Cardiol 1984; 53: 1529-31.

3 Dunn RF, Freedman B, Bailey IK, Uren RF, Kelly DT.

Dunn RF, Freedman B, Bailey IK, Uren RF, Kelly DT.
Location of coronary artery disease with exercise electroLocation of coronary artery disease with exercise electro-
cardiography: correlation with thallium-201 myocardial perfusion scanning. Am $\mathcal{f}$ Cardiol 1981; 48: 837-43.
4 Fuchs RM, Achuff SC, Grunwald L, Yin F, Griffith L. Electrocardiographic localization of coronary artery narrowings: studies during myocardial ischemia and infarction in patients with one-vessel disease. Circulation 1982; 66: $1168-76$.

5 Fox KM, Selwyn A, Oakley D, Shillingford JP. Relation between the precordial projection of ST segment changes after exercise and coronary angiographic findings. $A m \mathcal{F}$ Cardiol 1979; 44: 1068-79.

\title{
Warfarin potentiation with bezafibrate
}

\author{
Timothy RO Beringer
}

\author{
Summary \\ Two cases are described in which lack of \\ awareness of the potentiation of bezafi- \\ brate on warfarin was of clinical impor- \\ tance.
}

Keywords: bezafibrate, warfarin

Short- and long-term anticoagulant medication is widely and increasingly prescribed in a growing elderly population for the prevention and treatment of venous and arterial thromboembolism. Many of these patients have atherosclerosis and are also on lipid-lowering treatments. Increasing age and multiple medications are predisposing factors to adverse drug reactions. ${ }^{1}$ In addition, the list of drugs which may interact with warfarin continues to grow, with recent additions including the 4quinolone antibiotic ciprofloxacin, the selective serotonin re-uptake inhibitor group of antidepressants, and omeprazole.

\section{Case reports}

Case 1

A 65-year-old man presented with a fourday history of increasing weakness in the left arm and leg which had markedly worsened on the day of admission. He provided a history of childhood rheumatic fever, myocardial infarctions at the age of 53 and 58 years, and had undergone St Jude mitral valve replacement and coronary artery bypass grafting at the age of 64 years. $\mathrm{He}$ had subsequently received satisfactory therapeutic long-term anticoagulation with warfarin 4-5 mg daily. He was also receiving lipid-lowering therapy with bezafibrate $400 \mathrm{mg}$ daily, frusemide $40 \mathrm{mg}$, and amiloride hydrochloride $5 \mathrm{mg}$ daily.

Clinical examination confirmed the presence of left visual inattention, left upper motor neuron seventh nerve weakness and grade 4 power in the left arm and leg with preserved sensation. Computed tomography (CT) scan of brain demonstrated an area of reduced attenuation in the right frontal parietal region in keeping with infarction. His interna- tional normalised ratio (INR) was sub-therapeutic at 1.5 (reference range 2.0-3.5), blood urea was $8.2 \mathrm{mmol} / 1$ (reference range $3.3-8.8$ ) and serum creatinine $95 \mu \mathrm{mol} / 1$ (reference range 40-110). He was commenced on intravenous heparin $30000 \mathrm{IU}$ over 24 hours and his warfarin was continued. His INR failed to rise to therapeutic levels on $6 \mathrm{mg}$ rising to $7 \mathrm{mg}$ warfarin daily. Review of his medication revealed a low dose of bezafibrate of $200 \mathrm{mg}$ daily and institution of his previous dose of $400 \mathrm{mg}$ daily resulted in a therapeutic INR of 2.5 within 48 hours and withdrawal of his heparin. His limb weakness and visual inattention recovered, and he returned home walking independently. The patient then volunteered that during the week prior to admission he had not taken his normal dose of bezafibrate. He was unaware of the interaction between bezafibrate and warfarin. It is probable that the inadvertent reduction of bezafibrate had contributed to sub-therapeutic anticoagulation and the development of an embolic cerebrovascular event from a cardiac source.

Case 2

A 75-year-old woman was admitted after a fall resulting in a fracture of her right surgical neck of humerus and right neck of femur. She had a previous history of hypertension, heart failure, peripheral vascular disease, chronic obstructive airways disease and had been on treatment with warfarin from the age of 60 years following occlusion of a femoral-popliteal bypass. She had been commenced on lipidlowering therapy with bezafibrate $400 \mathrm{mg}$ daily in addition to a low-fat diet at the age of 69 years. Additional medication on admission included clonidine $100 \mu \mathrm{g}$ daily, amiloride $5 \mathrm{mg}$ daily, frusemide $40 \mathrm{mg}$ daily, salbutamol and ipratropium bromide inhalers, and her normal therapeutic dose of warfarin $1 \mathrm{mg}$ daily.

Initial investigations included a haemoglobin of $9.0 \mathrm{~g} / \mathrm{dl}$, urea of $9.7 \mathrm{mmol} / \mathrm{l}$, creatinine of $114 \mu \mathrm{mol} / 1$ and INR of 5.29. Her INR fell to a sub-therapeutic value of 1.2 five days following withdrawal of her oral warfarin. Following delayed surgery her warfarin was re-com- 
menced with a loading dose of $9 \mathrm{mg}$ and $6 \mathrm{mg}$ on successive days with her INR remaining elevated at $>4$ for the following four days. Review of her medication revealed that an incorrect dose of bezafibrate $400 \mathrm{mg}$ twice daily had been prescribed from admission. Following reduction to bezafibrate $400 \mathrm{mg}$ daily, therapeutic anticoagulation with warfarin $1 \mathrm{mg}$ daily was achieved. She made a satisfactory recovery, returning home independently mobile with a stick.

It is likely that the slow fall in her INR following admission was due to potentiation by the high dose of bezafibrate and the subsequent high INR recorded after warfarin was re-commenced with standard loading doses was similarly due to the high dose of bezafibrate.

\section{Discussion}

These cases highlight the clinical importance of the interaction between warfarin and bezafibrate and the lack of awareness by both patients and medical staff of the interaction. Increased awareness of this possibly life-threatening interaction is important as the coprescription of warfarin and bezafibrate medication is increasingly common in the setting of active treatment of thromboembolic disease together with lipid-lowering agents in atherosclerosis.

The potentiation of anticoagulants by lipidlowering agents has been described with clofibrate with androsterone, ${ }^{2}$ bezafibrate ${ }^{3,4}$

1 Hurwitz N. Predisposing factors in adverse reactions to drugs. $B M F$ 1969; 1: 536-9.

2 Oliver MF, Roberts SD, Hayes D, Pantridge JF, Suzman MM, Bersohn I. Effect of atromid and ethyl chloro phenoxyisobutyrate on anticoagulant requirements. Lancet 1963; i: $143-4$.

3 Blum A, Seligman H, Livneh A, Ezra I. Severe gastrointestinal bleeding induced by a probable hydroxycoumarin-bezafibrate interaction. Isr f Med $S c i$ 1992; 28: 47-9.

4 Zimmerman R, Ehlers W, Walter E, et al. The effect of bezafibrate in the fibrinolytic enzyme system and the drug interaction with racemic phenprocoumon. Atherosclerosis 1978; 29: $477-85$.

\section{Learning points}

- anticoagulant doses of warfarin should be reduced by a third to a half with the introduction of bezafibrate lipid-lowering therapy

- patients on warfarin and bezafibrate should be alerted to the importance of careful compliance with both medications to achieve safe and effective anticoagulation

- physicians caring for patients (especially the elderly) on warfarin and bezafibrate should be aware of the importance of checking compliance and current prescriptions of dosage, particularly on admission to hospital

and lovastatin. ${ }^{5}$ The exact mode of interaction between bezafibrate and warfarin is unclear. Bezafibrate is $95 \%$ protein-bound ${ }^{6}$ but rather than displacing warfarin from its binding site to albumin it may interact by increasing the affinity of the receptor sites for coumarins. ${ }^{4}$ As the fibrates increase the effect of oral anticoagulants, it is appropriate to reduce anticoagulant doses by a third to a half. ${ }^{7}$

These two cases highlight the clinical relevance of this interaction. Increased awareness is particularly important with the reduction in centralised hospital monitoring of anticoagulant therapy and the increase in general practitioner supervision of warfarin control. These cases were reported to the Committee on Safety of Medicines.

5 Iliadis EA, Konwinski MF. Lovastatin during warfarin therapy resulting in bleeding. Penn Med 1995; 98 (12): 31. 6 Lozada A, Dujovne LA. Drug interactions with fibric acids. Pharmacol Ther 1994; 63: 163-76.

7 Stockley IV. Drug interactions: a source book of adverse interactions, their mechanisms, clinical importance and management. Oxford: Blackwell, 1994. 